\title{
Essais
}

ESSAIS

Revue interdisciplinaire d'Humanités

$10 \mid 2016$

Faire-valoir et seconds couteaux

\section{Un braquage dans l'Histoire : la prise en otage de Sparte et d'Athènes par les universités allemandes et françaises}

Patrice Brun

\section{OpenEdition}

Journals

Édition électronique

URL : http://journals.openedition.org/essais/4156

DOI : $10.4000 /$ essais.4156

ISSN : 2276-0970

Éditeur

École doctorale Montaigne Humanités

Édition imprimée

Date de publication : 15 septembre 2016

Pagination : 165-182

ISBN : 978-2-9544269-9-0

ISSN : $2417-4211$

Référence électronique

Patrice Brun, « Un braquage dans l'Histoire : la prise en otage de Sparte et d'Athènes par les

universités allemandes et françaises », Essais [En ligne], 10 | 2016, mis en ligne le 15 octobre 2020,

consulté le 21 octobre 2020. URL : http://journals.openedition.org/essais/4156 ; DOI : https://doi.org/ 10.4000/essais. 4156 


\section{Un braquage dans l'Histoire : la prise en otage de Sparte et d'Athènes par les universités allemandes et françaises}

\section{Patrice Brun}

Nous savons tous que l'héritage des Grecs, tant au point de vue de la philosophie, de l'histoire, du théâtre, des arts, a depuis longtemps été revendiqué avec insistance par la culture occidentale. Dans ce cadre de pensée qui naît à la Renaissance et s'épanouit au XIX ${ }^{e}$ siècle avec la naissance des universités modernes, l'histoire grecque a longtemps servi de référence naturelle à des systèmes politiques ou philosophiques, et d'explication commode à une forme ou une autre de permanence des vertus et des tares morales de la nature humaine ${ }^{1}$. Et, dans cette optique, l'histoire grecque et singulièrement l'histoire aux temps classiques sous toutes ses formes et toutes ses sources ont donc été une base de réflexion majeure.

Mais une question méthodologique se pose alors : comment une période aussi éloignée que nous dans le temps, l'Antiquité, a-t-elle pu être utilisée pour nourrir des débats contemporains avec des arguments parfois très contradictoires ? Cela fait plusieurs décennies qu'ont été mises en exergue toutes les différences qui nous séparent des Grecs de l'Antiquité et la nécessité d'une mise à distance entre eux et nous ${ }^{2}$. Dans quelle mesure passe-t-on d'un actualisme raisonné, d'un comparatisme profitable à une "actualisation sauvage ", pour reprendre les mots de Pierre Vidal-Naquet ${ }^{3}$, un anachronisme consciemment ou inconsciemment perverti ? La question n'est pas simple à résoudre.

1 On consultera avec profit les actes d'un colloque édités par S. Caucanas, R. Cazals, P. Payen, Retrouver, imaginer, utiliser l'Antiquité, Toulouse, 2001, où sont étudiés nombre d'exemples d'utilisation et de détournement de l'histoire antique.

2 Cf. entre autres M.I. Finley, Democracy ancient and modern, Londres, 1973 (trad. fr. Démocratie antique et moderne, Paris, 1976) ; P. Veyne, Linventaire des différences, Paris, 1976 ; P. VidalNaquet, Les Grecs, les historiens, la démocratie, Paris, 2000 ; C. Ginzburg, Occhiaci di legno. Nove riflessioni sulla distanza, Milan, 1998 (trad. fr. À distance. Neuf essais sur le point de vue en histoire, Paris, 2001). Sur l'œuvre de ce dernier, cf. les contributions à elle consacrée par la revue Essais, sous le titre L'estrangement. Retour sur un thème de Carlo Ginzburg, S. Landi (éd.), hors série, 2013.

3 P. Vidal-Naquet, Les Grecs, les historiens, la démocratie, p. 25. 
Jacques Rancière et Nicole Loraux ont posé les jalons moins d'une (ré)habilitation de l'anachronisme que de son utilisation consciente et contrôlée pour permettre la connexion d'une ligne de temporalité à une autre ${ }^{4}$. Ainsi que l'exprime brutalement cette dernière, être historien, c'est assumer le risque de l'anachronisme, c'est presque le susciter en allant "du présent vers le passé avec des questions du présent pour revenir vers le présent, lesté de ce que l'on a compris du passé $"^{5}$.

C'est dans ce sens qu'il faut comprendre deux ouvrages, l'un oublié, l'autre bien connu, dont la conception et la rédaction eurent pour terreau la défaite de la France en 1940 et la naissance de la Révolution Nationale qui s'en suivit ${ }^{6}$. Les deux historiens, parvenus alors à leur maturité, s'interrogent ouvertement sur les rapprochements qu'ils voient entre des situations qu'ils jugent similaires - ou plutôt parallèles entre l'Athènes de 403 et la France de 1940.

Mais c'est tout autre chose dont il est question lorsque nous parlons de la manière dont les universitaires allemands ont utilisé la Sparte antique ou leurs homologues français l'Athènes de Démosthène. Comme on va le voir, c'est en conscience, par les mots employés, et plus encore par les buts poursuivis, qu'ils ont fait de une histoire contemporaine de combat sous le couvert d'une étude de la période antique. Ils ont fait de l'histoire un champ de bataille intellectuel au service de leur pays, je devrais dire, de leur patrie. C'est en cela qu'il est possible de parler d'une prise en otage de l'Antiquité, presque d'un braquage. Pour illustrer cette manière de faire de l'histoire, je vais utiliser deux exemples du traitement que l'on a fait subir - le mot n'est pas trop fort - aux deux cités les plus célèbres, Athènes et Sparte. Pour des motifs différents, l'université allemande pour Sparte, l'université française pour Athènes, ont utilisé, interprété, déformé à dessein l'histoire de ces cités et de leurs personnages les plus importants. Je vais donc convier ici non pas à une destruction, mais à une déconstruction des mythes de la Sparte de Lycurgue et de l'Athènes de Démosthène et il s'agira alors de comprendre la part de comparatisme et d'anachronisme qui existe dans ces prises de position. Sans négliger le fait que, rien, dans ces approches, n'est laissé au hasard : comme l'indique fort bien l'historien Pascal Payen, « dans la démarche historique, l'analogie n'est pas une donnée ou une évidence ; elle est une construction, une démarche de l'intellect, pour toujours mieux ajuster les rapports entre le présent et le passé ${ }^{7}$ ».

4 J. Rancière, "Le concept d'anachronisme et la vérité de l'historien ", L'Inactuel, 6, 1996, p. 53-68 ; N. Loraux, "Éloge de l'anachronisme en histoire ", Le Genre humain, 27, 1993, p. 23-39.

5 N. Loraux, art. cit., p. 26.

6 P. Jouguet, Révolution dans la défaite. Études athéniennes, Le Caire, 1942 ; J. Isaac, Les oligarques. Essai d'histoire partiale, Paris, 1946 (rédigé en 1942).

7 J.G. Droysen, Histoire de l'Hellénisme (trad. fr.), Paris, 2005, Introduction, p. 54. 


\section{L'Antiquité grecque et le nazisme}

Entendons-nous dès l'abord : tout historien du nazisme sait bien que ce régime n'est pas né subitement dans quelque arrière-boutique d'une brasserie munichoise, mais que son fonds idéologique racial puise ses origines dans un passé plus profond. Car il existe un Griechenmythos, que le non-germanique est tenu de traduire par le mythe grec allemand auquel un jeune historien, Anthony Andurand, a consacré un ouvrage ${ }^{8}$ : la pensée allemande, depuis la seconde moitié du XVIII siècle, a vénéré le passé grec, ce qui a abouti à une forme d'identification de l'Allemagne et de la Grèce'. Et, ainsi que le remarquait déjà Marc Bloch, à force de vénérer le passé, on est presque naturellement conduit à l'inventer ${ }^{10}$. Si le mythe est plus complexe qu'on ne pourrait le penser, car il a passablement évolué entre Goethe et Hitler, je vais en développer un axe essentiel, sans doute le plus connu, violemment anti-athénien et foncièrement favorable à Sparte et à la Macédoine, qui semble naître peu après les guerres napoléoniennes.

Dès lors, l'impression dominante est que l'émergence du sentiment national après les guerres napoléoniennes et la disparition de la symbolique du Reich millénaire, a suscité ses propres justifications historiques ${ }^{11}$. En 1824, un universitaire allemand, Karl Müller, publiait un livre intitulé Die Dorier, "Les Doriens ». Ce livre est à la base de l'idée qui a longtemps prévalu selon laquelle il existait dans le monde grec deux "races ", les Doriens, dont Sparte puis la Macédoine, étaient les plus beaux fleurons, et les Ioniens, qu'Athènes aurait représentés. Les Doriens, pour Karl Müller, sont des envahisseurs qui viennent du Nord et cette invasion serait la version historique de ce que le mythe désignait sous le nom de " retour des Héraclides ", des descendants d'Héraclès, qui auraient mis fin aux royaumes mycéniens vers les $\mathrm{XII}^{\mathrm{e}}-\mathrm{XI}^{\mathrm{e}}$ siècles avant notre ère ${ }^{12}$. Cette théorie a depuis été largement mise à mal sinon à néant par l'archéologie qui montre que les ruptures attendues d'une invasion violente sont invisibles à l'œil de l'archéologue, qui voit davantage à présent les continuités historiques et matérielles. Mais qu'importe ici.

On l'aura compris, les Doriens sont, pour Karl Müller, des Aryens - je rappelle que nous sommes ici un siècle avant la tentative de coup d'État d'Adolf Hitler, un siècle avant Mein Kampf. Dans son sillage, c'est toute une littérature à prétention historique ou anthropologique qui reprend cette

8 A. Andurand, Le mythe grec allemand. Histoire d'une affinité élective, Rennes, 2013.

9 A. Andurand, Le mythe grec allemand, p. 130.

10 M. Bloch, Apologie pour l'Histoire ou le métier d'historien, $2^{e}$ édition, Paris, 1993, p. 130. Cette réflexion vaut aussi pour les universitaires français face à un autre mythe, celui de l'Athènes de Démosthène (cf. infra).

11 Pour reprendre l'idée de P. Veyne, Comment on écrit l'histoire, Paris, 1971, p. 59-60.

12 Thucydide, I, 12, 3 ; Pausanias, IV, 3, 3. 
thèse, surtout en Allemagne mais pas uniquement, puisqu'elle est utilisée par Gobineau en France, par exemple. D'éminents professeurs de grec et d'histoire des universités allemandes durant tout le XIX ${ }^{\mathrm{e}}$ siècle et jusqu'en 1945 ont abondé dans cette voie, pro-spartiate et pro-macédonienne, rejetant dans la décadence le reste du monde grec.

Et c'est toute la cité d'Athènes, volontiers cosmopolite, marchande, démocratique, qui est en fait décriée par la science allemande, à l'exception peutêtre du temps de Périclès, car il était difficile de nier le Parthénon et l'ensemble des grands monuments de l'Acropole, modèle inavoué de la Welthauptstadt Germania dont Albert Speer avait conçu pour Hitler le projet ${ }^{13}$ : mais l'Athènes de Périclès, aux yeux des défenseurs de Sparte n'était pas une démocratie. C'était une monarchie déguisée, avec un chef bien identifié. Les universitaires allemands pouvaient respirer et un grand, un immense savant comme Ulrich Wilamowitz, dans un discours officiel en 1877 prend bien soin de distinguer la démocratie athénienne qu'il exècre, du Reich athénien - ce sont ses mots -, vraie tentative d'union de la Grèce par une cité impérialiste ${ }^{14}$. Helmut Berve, dont on verra un peu plus loin l'importance qui fut la sienne dans l'image de Sparte, rédigea une biographie de Périclès au tout début de la guerre, dans laquelle il fait de l'Athénien un parfait Aryen, Führer de la cité et dans laquelle il cultive un ensemble d'analogies artificielles destinées à rapprocher les figures de Périclès et d'Adolf Hitler ${ }^{15}$.

Mais à cette exception péricléenne près, c'est bien Sparte qui joue un rôle central dans la manière allemande de penser la Grèce, Sparte, considérée à partir du XIX ${ }^{\mathrm{e}}$ siècle comme une balise, un repère pour une Allemagne en voie d'unification ${ }^{16}$. Personne ne s'étonnera que les Spartiates soient représentés dans l'imaginaire allemand - et pas seulement nazi - comme de beaux Grecs blonds avec les yeux bleus et qu'ils ressemblent furieusement à une esthétique proche des Dieux $d u$ Stade de Leni Riefensthal ${ }^{17}$. Mais, à coup sûr, l'épanouissement de cette vision raciale et raciste date du Troisième Reich. Par idéologie ou par intérêt académique (il faut pouvoir obtenir et conserver

13 L.O. Larsson, Albert Speer : le plan de Berlin (1937-1943), Bruxelles, 1983 (2éd.).

14 J. Chapoutot, Le nazisme et l'Antiquité, Paris, 2008 [2012], p. 183.

15 H. Berve, Perikles, Leipzig, 1940, p. 21-25. Sur l'assimilation entre les deux hommes, voir V. Azoulay, Périclès. La démocratie athénienne à l'épreuve du grand homme, Paris, 2010, p. 226-229.

16 Outre le livre d'A. Andurand évoqué plus haut (note 8), les rapports entre Sparte et la pensée européenne dans sa globalité ont été analysés par E. Rawson, The Spartan Tradition in European Thought, Oxford, 1969 (rééd. 1991). Sparte et l'Allemagne, du XVIII' ${ }^{e}$ siècle jusqu'au nazisme, font l'objet du chapitre 19.

17 J. Bimbenet, Leni Riefensthal. La cinéaste d'Hitler, Paris, 2015, p. 142-145. La traduction française du film, "Olympie. La fête de la beauté ", oblitère le titre original en allemand, Olympia-Das Fest der Schönheit. Ce titre dit bien tout le lien que Leni Riefensthal faisait avec l'Antiquité. 
sa place), nombre d'universitaires nourris au lait de l'humanisme hellénique, ont alors sombré dans des théories raciales invraisemblables et assisté voire participé alors à une nazification consciente des lettres classiques, de l'histoire et de la philosophie antiques, sans que beaucoup de voix se fassent entendre contre ce dévoiement. Peu à peu, ce Nord imprécis a pris les contours d'une Germanie encore dans les limbes. La sophistique est ainsi pour le psychiatre et philosophe Kurt Hildenbrandt, auteur d'un ouvrage joliment intitulé Staat und Rasse $e^{18}$, "totalement étrangère à la pensée nordique ». Quant au stoïcisme "d'origine sémite " qui dénie l'appartenance à une cité pour privilégier la notion de "citoyen du monde ", il est la preuve d'une lourde décadence, amenant, je cite la traduction du passage d'un livre d'histoire générale, « le métissage des Grecs avec des peuples étrangers à la race nordique ». Quant au pauvre Socrate, annonciateur de ce stö̈cisme il est pour l'idéologue nazi Alfred Rosenberg, le «social-démocrate internationaliste de son temps » qui a sapé le fondement élitiste de l'inégalitarisme grec ${ }^{19}$.

Et Sparte est alors, comme en contrepoint de cette Athènes détestée, au cœur de cette invention de l'histoire. À la décharge des Allemands du temps, il faut dire que la naissance du «mythe spartiate » date de l'Antiquité, et que, tant Thucydide que Xénophon et surtout Plutarque y mirent leur patte. Le dernier surtout, dans sa pseudo-biographie du législateur Lycurgue, dont il disait par ailleurs que rien n'était certain dans sa vie, décrivit une Sparte largement fantasmée, idéalisée, composée de citoyens ayant suivi une éducation des plus rudes, durs avec leurs hilotes mais obéissants envers leurs magistrats, prêts à mourir plutôt que de reculer d'un pouce de terrain et ce, dès leur plus jeune âge, comme le rapporte Plutarque dans l'historiette bien connue du jeune garçon dévoré par un renard ${ }^{20}$. Dans ses Apophtegmes laconiens, il a d'autre part nourri le mythe du "laconisme", manière de parler peu mais avec beaucoup de sens, venu de Laconie, la région autour de Sparte, qui a accrédité l'image de Spartiates, taiseux, durs au mal, obéissants aux lois. Toutes choses développées dès l'Antiquité et qui, opposées à des Athéniens plus bavards et volontiers contestataires, firent de Sparte, dès l'avènement du Deuxième Reich qui ne vantait pas la démocratie parlementaire comme un modèle indépassable. On voit donc que ce mythe de Sparte avait des racines anciennes. Si l'on ajoute que les Spartiates parvinrent, à l'image de Guillaume Ir et de Bismarck pour l'Allemagne, à unifier le Péloponnèse dorien et même, à la fin de la guerre du Péloponnèse, l'ensemble du monde égéen sous sa coupe, on comprend comment et pourquoi le jeune Empire allemand a trouvé dans Sparte un exemple à suivre. Enfin, Sparte doit son aura dans la science germa-

18 K. Hildenbrandt, Staat und Rasse. Drei Vorträge, Breslau, 1928.

19 Sur tout cela, voir J. Chapoutot, Le nazisme et l'Antiquité, p. 306-307 ; p. 313-317.

20 Plutarque, Vie de Lycurgue, 18, 1. 
nique à ce racisme aryaniste né sous la plume de Müller et qui trouve des adeptes voyant en Sparte l'État « indo-germain » (Indogermanen, « indo-européen " en français) idéal, inégalitaire, eugéniste, militariste, expansionniste. On le comprend aisément, la voie était bien tracée pour le nazisme qui, loin là encore de créer une idéologie de toutes pièces, n'eut dans l'affaire qu'à cueillir des fruits déjà bien mûrs.

Presque tous les historiens ont adhéré à ces théories délirantes. L'exemple de Helmut Berve, bien connu des spécialistes de la Grèce antique est particulièrement édifiant ${ }^{21}$. Né en 1896 , issu de la grande formation allemande des études classiques et connaissant à merveille le latin et le grec, il publia en 1920, à l'âge de 24 ans seulement un mémoire sobrement intitulé Sparta, dans lequel, la plupart des poncifs "indo-européens " sur Sparte étaient déjà en place. Mais en 1937, adhérent depuis 1933 au NSDAP, nommé dans la foulée doyen de la faculté de Leipzig et désireux d'y demeurer et d'y faire carrière, il publie une seconde édition de sa monographie, beaucoup plus agressive et surtout tout à fait en phase avec le nouveau régime. Il y insiste sur l'éducation spartiate, cette agôgè très largement fantasmée ${ }^{22}$, qu'il était aisé, une fois déformée, de rapprocher des Hitlerjügend ${ }^{23}$. Cette éducation spartiate en classes d'âges, eugéniste, violente, formant à l'obéissance et à l'esprit de corps, était pour les nazis un champ presque inespéré.

Helmut Berve n'était pourtant pas un fanatique, mais un opportuniste qui accepta sans sourciller les thèses du $\mathrm{III}^{e}$ Reich pour poursuivre au mieux sa carrière : après une éclipse de quelques années à peine (il retrouva sa place à l'université en 1949), il est mort en 1979, entouré d'honneurs, après avoir poursuivi une vie universitaire des plus classiques sinon édifiante dans laquelle il gomma après 1945 toute allusion à ces écrits sulfureux. Il voulut oublier comment, en 1938, il prit la direction d'une revue vénérable, les Neue Jahrbücher für Antike, pour la transformer, 113 ans après sa naissance, en Neue Jahrbücher für Antike und Deutsche Bildung, dans laquelle la philologie n'occupe plus qu'une place dérisoire, remplacée par l'omniprésence des questions raciales ${ }^{24}$. On voit, à ce changement de nom que l'éducation, la formation d'un homme nouveau devait tirer de l'Antiquité grecque et de Sparte tout particulièrement des exemples destinés à montrer cette filiation entre

21 Sur le parcours de cet historien, S. Rebenich, "Alte Geschichte in Demokratie und Diktatur. Der Fall Helmut Berve ", Chiron, 31, 2001, p. 457-496.

22 Sur l'éducation spartiate dans son ensemble, cf. J. Ducat, Spartan Education, Cardiff, 2006.

23 La position de $\mathrm{H}$. Berve est à comparer avec le jugement qu'en donne P. Roussel dans un livre qui lui répond, Sparte, Paris, 1939, p. 160. Très hostile à Sparte, P. Roussel, sans doute pour railler les institutions spartiates, se plaît à parler de leur " caractère primitif " et à rappeler certaines données ethnographiques rapprochant le système militaire de Sparte à celui des Zoulous (cf. J. Sevry, Chaka, empereur des Zoulous : Histoire, mythes et légendes, Paris, 1991). Je ne suis pas certain qu'il s'agisse là d'un compliment.

24 In J. Chapoutot, Le nazisme et l'Antiquité, p. 190. 
Sparte et l'Allemagne nouvelle, entre Léonidas et Hitler en quelque sorte était naturelle. Et bien entendu, la guerre était au centre de ce rapprochement. La guerre, fleuron de Sparte. Les citoyens étaient, selon la légende, uniquement destinés à la guerre. Et cette légende, si j'en juge un film récent comme 300 de Zack Snyder ${ }^{25}$, perdure ou si je rappelle ces deux vers de Georges Brassens dans La guerre de 14-18:

Je sais que les guerriers de Sparte

Plantaient pas leurs épées dans l'eau...

Non qu'elle soit inventée de toutes pièces : Léonidas, dont je parlais plus haut, est bien mort aux Thermopyles après avoir résisté longtemps à une armée très supérieure en nombre. La phalange spartiate était redoutée et demeura invaincue jusqu'à la célèbre bataille de Leuctres en 371. Mais si l'armée de Sparte était fameuse, il ne faudrait pas oublier que toutes les cités grecques étaient modelées sur le principe premier de la guerre et de la défense du territoire. Les auteurs antiques ont largement vanté, pour des raisons souvent idéologiques, les qualités du soldat de Sparte. Mais on trouvait leur équivalent dans toutes les cités grecques.

Or, la bataille des Thermopyles est utilisée par l'idéologie nazie pour glorifier en quelque sorte la défaite de Stalingrad : comme les troupes spartiates face à l'avancée des Perses, l'armée allemande se serait sacrifiée pour empêcher l'ennemi soviétique d'envahir l'Allemagne. On ne s'étonnera donc pas que, en point d'orgue de cette maltraitance de l'Histoire, un manuel à destination des écoles intitulé "Sparta, der Lebenskampf einer nordischen Herrenschicht", "Sparte : la lutte pour la vie d'une élite nordique» (tout est dans le titre), paru en 1940, soit réédité en 1943 avec une préface d'Hermann Goering reprenant ce parallélisme fallacieux ${ }^{26} \ldots$

Vous l'aurez compris, malgré tout le salmigondis universitaire allemand, les Spartiates n'étaient pas un peuple nordique; ils n'étaient pas franchement blonds avec des yeux bleus, ne ressemblaient guère aux héros taillés dans le marbre par Arno Brekker, mais qu'importait finalement aux adeptes du grand Reich ! L'essentiel était de donner à l'Allemagne naissante des ancêtres intellectuels mieux présentables que des Germains peu ou prou vêtus de peaux de bêtes et de faire de ces derniers le ferment originel de cette Grèce blanche et blonde dont une branche de la famille avait un jour quitté les plaines du nord de l'Europe. Et, pour le Troisième Reich, l'occasion de trouver en un passé que l'on tordait avec allégresse, des traditions ancestrales qu'il fallait retrouver.

25 On se souvient moins d'un film de 1961, The 300 Spartans (en français La Bataille des Thermopyles), de Rudolph Maté, où les caractères des femmes et des hommes de Sparte, taillés dans le roc, définissent le Spartiate idéal, puisant chez Plutarque nombre de répliques définitives et anachroniques.

26 In J. Chapoutot, Le nazisme et l'Antiquité, p. 317-318 ; p. 558-561. 
Car il ne s'agissait pas ici de promouvoir un comparatisme historique fécond. À l'image de celui qu'un historien, Henri Jeanmaire, promut dans sa thèse, Couroi et Courètes $^{27}$, qui lui permit d'étudier les particularités de l'éducation et des rites d'initiation spartiates en les analysant au miroir de ce qui pouvait se pratiquer dans certaines sociétés africaines ${ }^{28}$.

Le mensonge, la volonté de tordre ce que l'on pouvait savoir de la réalité historique, dans le cas des nazis, étaient manifestes et l'anachronisme, qui nous apparaît au grand jour, était tout à fait nié. Tout au contraire, l'histoire officielle nazie insistait sur les similitudes, quitte bien entendu à les inventer de toutes pièces. Cette réécriture du passé ne leurrait sans doute pas même ceux qui le portaient. Mais, pour l'historien d'aujourd'hui, démasquer la tromperie ne suffit pas : il convient d'en découvrir les raisons ${ }^{29}$ et, dans le cas de Sparte et de l'Allemagne nazie, les raisons raciales apparaissent au grand jour.

\section{Athènes, Démosthène et l’Université française}

Face aux positions violemment anti-athéniennes et favorables à Sparte et à la Macédoine, la défense de la République française et de la démocratie fut organisée par l'Université depuis la place forte de la Sorbonne. Et, de ce point de vue, on peut dire que les historiens français ont tenu haut et fier le drapeau de la cause athénienne jusqu'à ce que l'on puisse parler, peut-être avec quelque exagération, d'une position "française " comme, pour l'autre camp, d'une position "germanique ». Athènes, sa littérature immense, ses monuments inégalables, ses statues d'une perfection absolue, ses hommes d'État prestigieux, de Thémistocle à Démosthène en passant par Périclès ou Alcibiade, est devenue le phare de la pensée, brillant haut et portant loin depuis l'université de la Sorbonne. Et pour cela, je vais prendre l'exemple de Démosthène, sans doute par paresse puisque j'ai publié il y a quelques mois de cela une biographie de l'orateur. C'est donc à partir de l'opposition entre Athènes et la Macédoine au IVe siècle que nous allons réfléchir.

On dit souvent - et non sans raison - que la guerre de 1870, la création de l'Empire allemand, en décidant l'unification politique des peuples germaniques sous la bannière prussienne, avait mis en exergue du côté des historiens allemands la Macédoine et son roi, Philippe II, le père d'Alexandre, déclenchant une riposte des historiens français, déniant au Macédonien le statut de Grec et trouvant en Démosthène un modèle de résistance.

27 H. Jeanmaire, Couroi et courètes. Essai sur l'éducation spartiate et sur les rites d'adolescence dans l'Antiquité hellénique, Lille, 1939.

28 H. Jeanmaire, Couroi et courètes, p. 156 : "Le choix d'exemples africains se recommande particulièrement lorsquill s'agit d'éclairer le passééloigné ou la préhistoire des sociétés méditerranéennes".

29 M. Bloch, Apologie, p. 129. 
Pourtant, le ministre de l'Instruction Publique de Napoléon III, Victor Duruy, avait, dès 1861, dans son Histoire grecque, affermi les bases déjà solides d'un soutien déclaré à l'orateur athénien. Mais c'est bien la guerre de 1870, la défaite des armées françaises et la perte de l'Alsace-Moselle qui a déclenché une violente riposte de l'historiographie française. En novembre 1870, en plein siège de Paris, paraissait dans la Revue des Deux Mondes, traditionnellement peu versée dans l'histoire ancienne, un article concernant la Macédoine de Philippe II au titre évocateur " Une Prusse dans l'Antiquité " ${ }^{30}$ et mettant au cœur du conflit francoallemand la lutte entre la Macédoine et Athènes qui, sur le plan historique, dit plus sur la France que sur le monde antique. D'un point de vue plus profond et moins immédiat, c'est Gustave Glotz (1862-1935) qui est indiscutablement le phare de la réflexion pro-démosthénienne en France. Pour comprendre son œuvre, il faut savoir que Glotz est né à Haguenau et qu'il quitta avec sa famille l'Alsace en 1871 pour rejoindre la «France de l'Intérieur ». Le souvenir de la défaite face aux troupes impériales prussiennes fut à coup sûr un élément constitutif de sa personnalité et de ses analyses historiques. Si ses premiers travaux sur la solidarité de la famille en Grèce, sur le travail, ressortissent surtout à la sociologie historique, ce sont deux œuvres fondamentales qui marquèrent longtemps - et qui marquent encore - les études démosthéniennes, La cité grecque, parue en 1928, et les tomes trois et quatre de l'Histoire grecque (1936 et 1938), deux œuvres posthumes. Dans ces ouvrages et notamment dans les deux derniers, se dessine la figure d'un Démosthène chez qui l'on ne décèle aucune faiblesse : excellence rhétorique, cohérence politique, noblesse des idées. Même partisan en 346 de la paix avec Philippe, il reste « au fond du cœur un adversaire irréconciliable de Philippe " et s'il se résout à la paix, il le fait « avec le sang-froid de l'homme d'État obligé de courber la tête sous une nécessité inéluctable »; s’il veut l'unité des Grecs, il "espérait y arriver par une évolution conforme au génie grec et en vue de sauver la liberté "; enfin, dans l'affaire d'Harpale qui détermina sa chute politique, affaire sordide de détournement d'une partie des sommes apportées par le trésorier d'Alexandre, " nul ne le crut vraiment coupable de vénalité " et Démosthène ne se rendit finalement coupable que d'un " prélèvement non autorisé » (sic). Enfin, la défaite finale n'est en rien à mettre au compte de la politique de Démosthène, mais du dèmos affaibli par « l'affaissement des caractères, la disparition du patriotisme et la politique du moindre effort » dont le peuple se rendit coupable ${ }^{31}$. Glotz, mort un an avant l'arrivée au pouvoir du Front Populaire, n'aurait pas été un franc partisan des congés payés.

30 A. Maury, Revue des Deux Mondes, nov. 1870, p. 405-428. Soulignons que cette identification était parfaitement assumée du côté allemand - on devrait dire du côté prussien - dès 1870 : A. Andurand, Le mythe grec allemand, p. 194-197.

31 Ses qualités d'orateur, Histoire grecque, III, p. 251-252; homme d'État, toujours adversaire de Philippe : p. 292, 299 ; option panhellénique : p. 377 ; l'affaire d'Harpale : Histoire grecque, IV, p. 216. 
À l'inverse, les adversaires de Démosthène ne trouvent jamais grâce à ses yeux : Gustave Glotz stigmatise " les cantilènes endormantes d'Isocrate » [qui], " après s'être égosillé pendant cinquante ans, finit en Chantecler. Il meurt convaincu qu'il a fait lever le soleil ». Eschine est étrillé : acteur de troisième ordre, orateur quelconque, il « n'a pas l'étoffe d'un homme d'État " et auprès de lui, " on se sent vite en présence d'une intelligence et d'une âme médiocres ». Philippe de Macédoine n'agit que par corruption, sa diplomatie est " cauteleuse ", ses ambassadeurs adoptent un " ton papelard ". Les alliés de Philippe sont " asservis au tyran ». Toutes ces citations reprennent en fait mot pour mot, sans aucun recul, les diatribes et anathèmes de Démosthène. Ce manichéisme fut, avec quelques nuances plus ou moins excessives, celui de la quasi-totalité de l'Université française dans l'entre-deux-guerres mais encore bien après, à de rares exceptions près.

Paul Cloché qui, à bien des égards fut le continuateur de la pensée de Gustave Glotz dans la manière de penser la Grèce et Athènes, suivit la même voie dans trois ouvrages importants ${ }^{32}$. Démosthène possède ainsi " un très vif souci de la grandeur et de la sécurité nationales "; ses accusateurs dans l'affaire d'Harpale ont été « incapables d'apporter une démonstration directe et rigoureuse de la culpabilité de leur ennemi ". Le comportement d'Eschine est vilipendé et, à propos de l'ambassade de 346 qui devait aboutir à la paix de Philocratès, Paul Cloché se sent autorisé à demander : « que valent ces explications d'Eschine en face des allégations si précises de Démosthène" ? Il est d'ailleurs d'une "vanité débordante " et s'il n'est pas forcément corrompu, "il a agi avec une légèreté puérile, indigne d'un homme d'État et singulièrement périlleuse $"^{33}$. Sans poursuivre de trop fastidieuses énumérations, on pourrait encore citer dans cette lignée contemporaine Jean Luccioni, Georges Mathieu, Jean Hatzfeld ou Gaston Colin, ce dernier avouant de façon naïve et touchante rechercher à tout prix l'innocence de Démosthène dans "l'affaire d'Harpale "34. Il y a certes des nuances dans les propos des uns et des autres. Mais la « ligne éditoriale » générale ne souffre guère de ces quelques remarques et verse souvent dans une lourde exagération, y compris dans l'étude de son talent oratoire, qu'il serait oiseux et infructueux de rapporter en détail ${ }^{35}$. Tous ont en commun une méthode historique consistant à lire l'histoire grecque des années 355-322 en suivant la

32 La politique étrangère d'Athènes, Paris, 1934 ; Démosthènes et la fin de la démocratie athénienne, Paris, 1937 (2e éd. 1957) ; Philippe de Macédoine : un fondateur d'empire, Saint-Étienne, 1955.

33 La grandeur : Démosthènes, p. 67 ; l'affaire d'Harpale : p. 287 ; les allégations d'Eschine : p. 114 ; sa vanité : p. 127.

34 G. Cousin, ibid., p. 228 : "S’ensuit-il que nous soyons tenus de considérer Démosthène comme tombé, sur la fin de sa vie comme tombé, sur la fin de sa vie, par amour du lucre, au rang d'un Démade ou d'un Aristogiton ? J'en conviens, cette pensée me serait pénible ; j'ai cherché, je cherche encore le moyen d'y échapper ".

35 Un seul exemple, celui de P. Orsini, éditeur des Plaidoyers politiques de Démosthène dans la CUF (t. 1, p. LX) à propos des trois premiers discours de l'orateur : « cette richesse de moyens, mi-étudiés, mi-instinctifs confine, dès le début, au génie ". 
chronologie des discours de Démosthène, source essentielle certes, mais très rarement critiquée ou peu mise en concurrence avec des sources rhétoriques adverses ou avec des inscriptions, pourtant nombreuses à avoir été conservées.

Mais ce que Gustave Glotz et ses épigones défendent, au travers de Démosthène, c'est évidemment la démocratie, la République Française, face à l'Empire allemand, aisément reconnaissable derrière le voile transparent qui recouvre le visage de Philippe de Macédoine. Le passage cité plus haut, accusant les Athéniens du temps d'être responsables de "l'affaissement des caractères, [de] la disparition du patriotisme et [de] la politique du moindre effort » ressemble ainsi trait pour trait à des leitmotiv des années 30 en France. Et Démosthène apparaît comme celui qui avertit ses concitoyens de la menace, nouveau Clemenceau en quelque sorte, Clemenceau qui publia peu avant sa mort, en 1926, une rapide biographie de l'orateur dans laquelle il n'est pas difficile de retrouver les traces de son combat politique ${ }^{36}$.

"En face », de l'autre côté du Rhin, la vision est tout autre comme bien l'on pense : pour Ulrich Kahrstedt, dont la carrière scientifique s'étendit sur plus de 50 années (1910-1962) dans un essai sur la politique extérieure des cités grecques, Démosthène n'est rien d'autre qu'un agent stipendié du roi de Perse, désireux d'empêcher à tout prix l'invasion de ses terres par les Macédoniens ${ }^{37}$. À peu près tous les épisodes de la vie politique de Démosthène, tous les mouvements de troupes athéniennes seraient, selon lui, dictés par cette obsession - laquelle semble être surtout celle de son auteur, lequel ne cache d'ailleurs pas un aspect racial à sa réflexion lorsque, non sans mépris, il parle d'une alliance de fait entre les Athéniens et les "Asiates " (Asiaten), terme dont on relèvera aisément le mépris qu'il comporte. Nous ne sommes là qu'en 1910. Et plus généralement, le principe même démocratique est mis en cause par Engelbert Drerup, qui, dans un ouvrage au titre évocateur et publié en pleine Première Guerre Mondiale (Aus einer Advokatenrepublik «À propos d'une République d'avocats »), qualifié par lui-même de "livre de guerre " (Kriegsbuch), défend en 1916 la thèse d'un Démosthène, tout empli de " fanatisme politique " prêt à tous les moyens pour abattre la Macédoine et pour cela vendu aux intérêts perses, en y ajoutant des réflexions morales hostiles à l'orateur qu'il estimait plein de bassesse, égoïste, sans idéal. Il honnit par la même occasion les principes démocratiques des échanges rhétoriques athéniens (et, par la même occasion, les parlementaires britanniques et français) et vante au contraire les mérites du " militarisme " macédonien (et allemand) en mettant en balance de manière forcée, je traduis, "un souverain qui a converti le monde à la civilisation d'Homère et un parlementaire démocratico-républicain aux vues étroites et égoïstes ${ }^{38}$.

36 G. Clemenceau, Démosthène, Paris, 1926.

37 U. Kahrstedt, Forschungen zur Geschichte des ausgehenden fünften und des vierten Jahrhunderts, Berlin, 1910.

38 E. Drerup, Aus einer Advokatenrepublik, Paderborn, 1916, p. 148. 
Sont-ce les guerres franco-allemandes qui sont seules à l'origine de ces oppositions intellectuelles très fortes ? Il ne faudrait pas donner une image trop caricaturale et trop "nationale " à cette opposition qui se résumerait à un heurt entre une Allemagne portée à aduler Philippe et une France canonisant Démosthène. En réalité, cette dichotomie dans l'analyse est tout autant idéologique que nationale même si, on en conviendra, entre 1870 et 1939, celle-ci recoupe assez bien les frontières que les Vosges ou le Rhin séparent. Mais la question se pose pour nous en ces termes : où est le "vrai » Démosthène dans ces propos croisés à ce point déformés par l'esprit du temps, si tant est qu'il soit possible de le mettre au jour?

Sans vouloir ici découvrir la Sparte authentique ni le véritable Démosthène, on doit se poser la question centrale de mon intervention : pourquoi ce lien entre Histoire grecque et histoire contemporaine ${ }^{39}$ ? Pourquoi l'Histoire grecque a-t-elle à ce point attisé les convoitises des historiens et des idéologies ? Pourquoi Sparte a-t-elle été utilisée à ce point ? Pourquoi Démosthène et, plus généralement Athènes et ses institutions ont-ils été biaisés par des réflexions très contemporaines? Et pourquoi ai-je donc donné en titre de cette conférence celui, un brin provocateur, de "braquage " ? C'est sur ce point que je voudrais à présent insister en essayant de comparer la démocratie antique et la démocratie moderne. Car, il faut bien le dire, si Sparte est aujourd'hui l'objet d'études scientifiques apaisées, les errements idéologiques du III ${ }^{e}$ Reich l'ont condamnée à ne plus être un modèle.

Sparte et Démosthène. Victimes tous les deux d'un hold-up de leur réalité historique par la postérité. Mais plus généralement, on se rend compte, au fil de l'analyse comparée des textes antiques et des représentations contemporaines, que c'est la Grèce antique, dans sa globalité qui a été annexée par la postérité occidentale. On l'a déjà vu, avec le mythe grec allemand, mais l'affaire est plus générale : en 1964, un écrivain français, Thierry Maulnier, qui s'était illustré entre 1932 et 1944 par sa participation à des revues fascisantes et qui termina sa vie comme membre de l'Académie Française, publia un livre de réflexions, Cette Grèce où nous sommes nés, dont le titre seul indique l'orientation : nos racines sont moins judéo-chrétiennes que gréco-romaines, et plus grecques que romaines. Et il puise dans l'art, la littérature sous toutes ses formes, du théâtre à l'histoire en passant par la rhétorique, le théâtre et la philosophie de quoi alimenter l'obsession antisémite de ses jeunes années. La Grèce méritait-elle cette nouvelle prise en otage ? Un ouvrage récent, Le mythe de la Grèce blanche, signé d'un archéologue, Philippe Jockey, a démonté le fil de cette pensée intrusive à partir de l'exemple artistique : quand on voit la blancheur du Parthénon, de la Vénus de Milo, de la Victoire de Samothrace

39 Le parcours de Pierre Vidal-Naquet, historien de l'histoire immédiate (L'affaire Audin, Paris, 1958) et de l'antiquité grecque symbolise ce rapprochement. 
et d'autres merveilles de l'art grec taillés dans le marbre immaculé de Paros ou du Pentélique, on se plaît à imaginer des sculptures d'un albe éclatant de lumière. Cette idée du "blanc absolu " n'est évidemment pas neutre et elle nourrit des fantasmes raciaux moins avoués, mais tout aussi prégnants que ceux qui prévalaient il y a moins d'un siècle. Sauf que... Sauf que les études chimiques les plus récentes montrent que toutes, TOUTES les sculptures, TOUS les temples, en premier lieu le Parthénon, étaient d'une polychromie éclatante, les sculptures, surtout les plus fameuses étant de surcroît dorées à la feuille. Il n'y a pas de Grèce blanche, pas davantage qu'il n'y avait de Spartiate blond ni de Démosthène seul contre tous. Mais que reste-t-il alors de la Grèce antique ? Si le mythe s'effondre, la réalité subsiste, au travers de ce que nous pouvons savoir de la démocratie et j'en terminerai par là, dans une forme de comparaison raisonnée entre le système démocratique antique et le nôtre.

\section{Démocratie antique et démocratie moderne}

Lorsque je parle de démocratie moderne, je veux évidemment parler des démocraties d'aujourd'hui dites occidentales et laisserai de côté tant les " démocraties populaires » de l'Europe de l'Est et de son avatar actuel, la si pittoresque République Populaire Démocratique de Corée, que la « démocratie dirigée » chère au général Pinochet. C'est-à-dire que je parle des démocraties que l'on appelle " représentatives " ou "parlementaires ", qui désignent sans tricherie organisée des hommes ou des femmes pour représenter l'ensemble de la population civique du pays, population évidemment incapable de prétendre à une forme ou une autre de " démocratie directe ". Et c'est là le premier point que je voudrais développer en attaquant le sujet par rapport à des expériences personnelles.

Il est toujours aisé, lorsque l'on est historien, de décerner bons et mauvais points aux personnages du passé que l'on étudie, d'affirmer qu'ils auraient dû faire ceci et cela ; facile aussi - et je viens de le faire ici - de critiquer les prédécesseurs qui ont tenté de définir ce qu'était Sparte ou d'affiner le portrait de Démosthène. Mais ce n'est pas parce que l'on se croit averti du piège que l'on n'y tombe pas soi-même. Helmut Berve ou Gustave Glotz ont été victimes en grande partie de la période dans laquelle ils ont vécu, de l'atmosphère intellectuelle et politique qui agitait leurs pays respectifs. Qu'aurais-je fait, qu'aurions nous fait en leurs lieux et temps? Lorsque je me retourne sur moi-même, je ne peux que m'interroger : je n'avais pas quinze ans en mai 68 et toute mon adolescence a baigné dans un débat d'idées tournant autour des figures intellectuelles de Raymond Aron et de Jean-Paul Sartre et des questions autour du sens et du contenu à donner au mot démocratie. Questions qui amenaient à peser les qualités et défauts respectifs de la démocratie directe, de celle des soviets ou de la démocratie représentative. Bien sûr que j'en ai été "victime » 
et que des séquelles de ces débats hantent, volens nolens, encore mon esprit ! Et d'autre part, je suis issu d'un milieu familial pour qui le grec était une terre totalement inconnue et le latin limité à quelques formules magiques prononcées par le prêtre. Personne ne m'a nourri au lait des textes anciens : si j'ai appris le latin au collège comme tout le monde ou presque le faisait alors, je n'ai commencé à apprendre le grec qu'à l'université, dans l'équivalent de la L3 à présent. C'est dire que je n'ai abordé les textes grecs que bardé d'un esprit critique bien plus avancé que si je n'avais débuté cet enseignement sept ou huit ans avant. Aucun de mes enseignants ne m'a jamais parlé de la beauté de la langue, de la fluidité du style de Démosthène et c'est sans doute cela qui fait que je suis plutôt critique vis-à-vis du personnage historique de Démosthène, parce que je me suis éduqué au grec avec la passion de l'historien du politique bien plus qu'avec celle, tout aussi noble d'ailleurs, du linguiste.

Mais, pour ce qui me concerne, cela va plus loin encore. En tant que Président d'université durant la dernière grande émotion universitaire en 2009, j'ai vécu de très près ces expériences de "démocratie directe » ou prétendues telles que sont les assemblées générales. Présent à toutes, j'ai pris la parole quand je le jugeais nécessaire, mais j'ai surtout observé avec certes un œeil de Président, et tout autant avec celui de l'historien de la Grèce ancienne que je suis ou essaie d'être. Et je me disais que, au total, ces assemblées qui offraient en principe l'isègoria, l'égalité de la parole à tous - encore qu'il est difficile d'imaginer que trois ou quatre mille étudiants s'expriment successivement devaient assez ressembler à ce qui se passait à l'assemblée du peuple si l'on en croit les témoignages qui nous restent. Ces assemblées générales duraient toute la journée ou presque et c'étaient toujours les mêmes qui prenaient la parole, affirmant avec autorité parler au nom de tous ou plus exactement pour l'aile marchante de la petite société étudiante. C'est que s'exprimer devant une foule compacte de plusieurs milliers de personnes nécessite un entraînement que des militants formés à la rude école de « l'agit-prop » ont plus de capacité à suivre, à l'image des hommes tels Démosthène, formés à la pratique de la rhétorique par des maîtres grassement payés. Aussi bien, la " démocratie directe » est-elle loin de fournir l'image d'une égalité autre que de façade.

Et c'est souvent le reproche qui a été fait à la démocratie athénienne : une égalité qui n'en était pas une. Ce reproche date de l'Antiquité elle-même, mais elle se situait sur un plan différent, parce que les philosophes qui ont critiqué la démocratie, soit dans ses excès comme Aristote, soit dans sa structure même comme Platon, avaient du mal à accepter que des citoyens sans éducation - ou plus exactement sans l'éducation philosophique à laquelle ils destinaient les hautes sphères de la société - et sans fortune pussent diriger la cité du seul fait de leur nombre par rapport aux " nobles ", aux "bien-nés " comme on disait plutôt à l'époque (eugeneis). Aujourd'hui, les critiques que des historiens, surtout américains et de conviction démocrate, au sens que l'on donne à ce mot aux États-Unis, portent sur la démocratie sont d'un autre type. 
Bien entendu, la question de l'esclavage, de la place des femmes, de la non-intégration pour ainsi dire définitive des étrangers, pèse d'un poids très lourd, mais ce sont des thèmes si visibles et connus depuis si longtemps qu'il n'est pas nécessaire d'y insister, sauf pour signaler que certains défenseurs à outrance de la démocratie athénienne imaginaient que l'esclavage était, au $\mathrm{IV}^{\mathrm{e}}$ siècle, en voie de disparition à Athènes et que seule la défaite face aux Macédoniens a empêché cette libération grandiose. De fait, il est inutile et à coup sûr anachronique d'affirmer en se drapant dans sa dignité que la démocratie athénienne n'en était pas une puisque les femmes n'avaient aucun pouvoir et que l'esclavage était très important. C'est oublier qu'en France, les femmes n'ont accédé au droit de vote qu'en 1946 et les femmes mariées au droit d'ouvrir un compte bancaire à leur nom en 1975 seulement. C'est oublier que la première démocratie moderne, celle des États-Unis, s'accommoda fort bien de la traite jusqu'en 1820 et de l'esclavage lui-même jusqu'à la fin de la guerre de Sécession. Hors sujet, donc.

Mais en réalité, les critiques les plus marquées sont aujourd'hui liées au phénomène de l'impérialisme athénien. Il fallut attendre les années soixante pour que, la décolonisation aidant, certains historiens perçoivent l'impérialisme athénien sur les alliés de la Ligue de Délos à l'aune de la colonisation occidentale en Afrique. Tant que les sociétés occidentales étaient persuadées de la légitimité de la colonisation, l'impérialisme athénien ne posait pas de problème d'ordre éthique. Mais avec le phénomène de décolonisation et surtout avec les guerres coloniales et post-coloniales, la situation a changé. C’est avant tout aux États-Unis, très fortement marqués par la guerre du Viet-Nam que le phénomène a pris le plus d'ampleur. Je m'arrêterai sur deux historiens de l'Antiquité.

Le premier s'appelle Lawrence Tritle. Né en 1946, c’est un vétéran du VietNam, devenu spécialiste de la politique athénienne. En 2000, il a fait paraître un travail tout à fait étonnant, From Melos to My Lai ${ }^{40}$. Mélos, île athénienne vaincue, réduite en esclavage et massacrée par les Athéniens en 416. My Lai, village vietnamien, où, en 1968, un corps d'armée américain massacra des centaines de villageois, pour l'essentiel des femmes et des enfants. Au-delà de la quasi-homophonie des mots, Lawrence Tritle, qui n'avait pas pris part à ce massacre, mettait pour la première fois en regard les deux démocraties, l'athénienne et l'américaine, dans ce qu'elles avaient de pire, tout cela au nom même de la démocratie ou de l'idée qu'elles s'en faisaient.

Le second historien est Lorens Jr Samons. Né en 1965, il est de la génération d'après, celle qui n'a connu du Viet-Nam que Platoon ou Rambo. Mais il est de celle du 11 Septembre, de l'invasion de l'Irak et de l'Afghanistan, au

40 L. Tritle, From Melos to My Lai. A Study in Violence, Culture and Social Survival, Londres-NewYork, 2000. 
nom des valeurs démocratiques. En 2007, il publia un livre au titre évocateur : What's Wrong with Democracy? From Athenian Practice to American Worship ${ }^{41}$, "Qu'est-ce qui ne va pas avec la démocratie ? De la pratique athénienne au culte américain ". Il part de la situation dans son propre pays au début du $\mathrm{XXI}^{\mathrm{e}}$ siècle : la démocratie est devenue une icône, elle bénéficie d'un véritable culte et on imagine que, seule, elle peut apporter le bonheur aux peuples. Il faut donc l'imposer, fût-ce par les armes les plus modernes et les plus mortelles. C'est-à-dire imposer des élections, quel que soit le degré de violence qui subsiste, en sortir un gouvernement "représentatif » ou prétendu tel. Et rien d'autre... sauf pour arriver à ce but ultime imposer des humiliations, perpétrer des massacres, emprisonner en masse comme à Guantanamo. Les images de la prison de Bagdad connue sous le nom d'Abou Ghraïb résonnent encore dans la tête de Lorens Samons. Et lui aussi fait le parallèle avec Mélos, mais aussi d'autres massacres commis par les Athéniens au nom de la « démocratie » et surtout au nom de l'idée qu'ils s'en faisaient.

On le voit avec ces deux exemples, la démocratie athénienne, modèle périmé s'il en est, et l'histoire grecque en général nous parlent encore. Mais à certaines conditions.

Il convient tout d'abord de ne pas idéaliser la démocratie athénienne qui s'est souvent auto-célébrée, que ce soit Périclès dans ce célèbre passage connu sous le nom de "l'oraison funèbre ", ou Démosthène dans la plupart de ses discours, lui qui vanta jusqu'à l'infini la douceur du régime démocratique. Les remarques que j'ai faites sur les massacres perpétrés par la démocratie athénienne, en son nom ou plutôt dans l'intérêt des Athéniens eux-mêmes en disent assez sur l'absence nécessaire de toute idéalisation. De la même manière, l'acte de monter sur l'Acropole ne devrait pas être assimilé à une idée de pèlerinage, quand bien même des guides grecs payés pour cela voudraient nous obliger à le croire.

Ensuite, nous devons admettre, ainsi que j’ai essayé de le montrer, que le modèle démocratique athénien n'est pas l'ancêtre de notre système actuel pour une raison simple. Pour les Grecs, les droits et les devoirs d'une personne dépendaient avant toute chose de leur statut. Selon que l'on était Grec ou barbare, homme ou femme, enfant ou adulte, Athénien ou Mélien (par exemple), les contours de la liberté individuelle étaient largement modifiés. Notre modèle actuel est tout autre, fondé sur les Droits de l'Homme qui ont, entre 1679 et 1791, fourni des principes qui règlementent non pas le système institutionnel, mais la liberté individuelle. En 1679, le Parlement anglais vote un texte intitulé : «Habeas corpus ad subjiciendum », "Que tu aies ton corps à ta disposition (pour le produire devant le tribunal) ", plus connu sous le

41 L. Samons, What's Wrong with Democracy? From Athenian Practice to American Worship, Berkeley, 2004 . 
nom de Habeas Corpus, qui énonce une liberté fondamentale, celle de ne pas être emprisonné sans jugement. Dix ans plus tard, en 1689, à l'issue de la Glorieuse Révolution, le Bill of Rights affirme des droits positifs que les citoyens et/ou les résidents d'un pays en monarchie constitutionnelle devaient avoir et expose également certaines exigences constitutionnelles : toute action de la part du monarque exige l'assentiment du gouvernement tel qu'il est représenté par le Parlement. Un siècle après, en 1789, c'est en France la Déclaration des Droits de l'Homme et du Citoyen sur laquelle il n'est pas nécessaire de s'appesantir. Deux ans plus tard, en 1791, le United States Bill of Rights limite les pouvoirs du gouvernement fédéral et garantit les libertés de presse, de parole, de religion, de réunion, le droit de porter des armes, et le droit de propriété.

Ce sont ces textes fondateurs qui définissent aujourd'hui notre conception de la démocratie, dans laquelle les libertés individuelles jouent un rôle de premier plan. Ces aspects-là étaient strictement inconnus de la Grèce antique, dans laquelle c'est le groupe auquel appartient l'individu qui définit ses droits ou son absence totale de droits. Et on en revient à cette séparation citoyens / non-citoyens, qui permettait par exemple, en toute légalité, sans loi d'exception aucune, de torturer un esclave pour obtenir en justice un témoignage quelconque ; même s'il n'était accusé de rien, mais qu'il avait été témoin de quelque affaire. Décidément, le monde antique est bien loin de nous et il faut en manier l'exemplarité avec une grande prudence.

Je ne vous ai dit qui était Démosthène ni quelle était Sparte. Ce n'était pas mon but ici. J'ai tenté de démêler les fils de la connaissance pure de ceux de l'apprentissage de cette connaissance. De tenter de montrer, pour autant que notre mémoire personnelle nous soit fidèle, ce qui ressortit à notre vécu, lié à la fois à notre propre intellect qu'à notre environnement proche mais aussi national sinon international. Et, s'agissant au final des exemples extraits de l'Antiquité que j'ai choisis, on peut penser que les historiens du temps avaient pleine conscience qu'en vantant - en les déformant avec brutalité - les mérites de Sparte, en soulignant la grandeur de la démocratie athénienne sous Démosthène - en la présentant sous des jours exagérément roses - ils faisaient en fait de l'histoire contemporaine. Et ces déformations volontaires, j'espère vous en avoir convaincus, sont bien un délit intellectuel, un vol avec effraction de l'histoire antique, un braquage d'une civilisation qui ne le demandait pas.

Patrice Brun UMR 5607 AUSONIUS Université Michel de Montaigne Patrice.Brun@u-bordeaux-montaigne.fr 


\section{Keywords}

Ancient Greece, Sparta, Athens, historiographic currents.

Mots-clés

Antiquité grecque, Sparte, Athènes, courants historiographiques. 